\title{
Functional and Structural Connectivity Patterns Associated with Clinical Outcomes in Deep Brain Stimulation of the Globus Pallidus Internus for Generalized Dystonia
}

\author{
(D). Okromelidze, (D). Tsuboi, (D).S. Eisinger, (D) M.R. Burns, (D). Charbel, (D). Rana, (D).S. Grewal, (D).-Q. Lu, (D). Almeida,
}

(D) K.D. Foote, (D) M.S. Okun, and (D)E.H. Middlebrooks

\begin{abstract}
BACKGROUND AND PURPOSE: Deep brain stimulation is a well-established treatment for generalized dystonia, but outcomes remain variable. Establishment of an imaging marker to guide device targeting and programming could possibly impact the efficacy of deep brain stimulation in dystonia, particularly in the absence of acute clinical markers to indicate benefit. We hypothesize that the stimulation-based functional and structural connectivity using resting-state fMRI and DTI can predict therapeutic outcomes in patients with generalized dystonia and deep brain stimulation.
\end{abstract}

MATERIALS AND METHODS: We performed a retrospective analysis of 39 patients with inherited or idiopathic-isolated generalized dystonia who underwent bilateral globus pallidus internus deep brain stimulation. After electrode localization, the volumes of tissue activated were modeled and used as seed regions for functional and structural connectivity measures using a normative data base. Resulting connectivity maps were correlated with postoperative improvement in the Unified Dystonia Rating Scale score.

RESULTS: Structural connectivity between the volumes of tissue activated and the primary sensorimotor cortex was correlated with Unified Dystonia Rating Scale improvement, while more anterior prefrontal connectivity was inversely correlated with Unified Dystonia Rating Scale improvement. Functional connectivity between the volumes of tissue activated and primary sensorimotor regions, motor thalamus, and cerebellum was most correlated with Unified Dystonia Rating Scale improvement; however, an inverse correlation with Unified Dystonia Rating Scale improvement was seen in the supplemental motor area and premotor cortex.

CONCLUSIONS: Functional and structural connectivity with multiple nodes of the motor network is associated with motor improvement in patients with generalized dystonia undergoing deep brain stimulation. Results from this study may serve as a basis for future development of clinical markers to guide deep brain stimulation targeting and programming in dystonia.

ABBREVIATIONS: $\mathrm{ACPC}=$ anterior/posterior commissure; $\mathrm{DBS}=$ deep brain stimulation; $\mathrm{GPi}=$ globus pallidus internus; $\mathrm{MNI}=$ Montreal Neurological Institute; UDRS = Unified Dystonia Rating Scale; VTA = volume of tissue activated

D ystonia is a debilitating movement disorder with the primary features being intermittent or sustained muscle co-contractions with abnormal postures and/or repetitive movements. ${ }^{1}$ Of the different recognized forms of dystonia, generalized dystonia affecting the trunk and a minimum of 2 other body regions can be particularly debilitating. ${ }^{2}$ Oral medications such as anticholinergics, benzodiazepines, or muscle relaxants can be attempted but are often poorly tolerated due to adverse effects such as

Received October 22, 2019; accepted after revision January 7, 2020.

From the Departments of Radiology (L.O., C.-Q.L., E.H.M.) and Neurosurgery (S.S.G., E.H.M.), Mayo Clinic, Jacksonville, Florida; Department of Neurology (T.T., R.S.E., M.R.B., L.A., K.D.F., M.S.O.), Norman Fixel Institute for Neurological Diseases, Department of Neurosurgery (K.D.F.), and J. Crayton Pruitt Family Department of Biomedical Engineering (M.C.), University of Florida, Gainesville, Florida; and Institute of Medical Psychology and Behavioural Neurobiology (M.R.), University of Tübingen, Tübingen, Germany.

L. Okromelidze and T. Tsuboi contributed equally to this work.

Paper previously presented in abstract form at: Annual Meeting of the Radiological Society of North America, December 1-6, 2019; Chicago, Illinois. sedation and cognitive dysfunction. Deep brain stimulation (DBS) is a well-established treatment for generalized forms of dystonia. ${ }^{3,4}$ Although the mechanism of action is not wellunderstood, globus pallidus internus (GPi) DBS has been one of the most widely adopted targets for the treatment of generalized and cervical dystonia. ${ }^{5}$ Despite the increasing use of DBS, patient outcomes have been highly variable, with multiple factors potentially playing a role, including phenomenology and body parts affected as well as a delayed response to the settings implemented. ${ }^{3,4,6,7}$

Please address correspondence to Erik H. Middlebrooks, MD, Departments of Radiology and Neurosurgery, Mayo Clinic, 4500 San Pablo Rd, Jacksonville, FL 32224; e-mail: middlebrooks.erik@mayo.edu; @EMiddlebrooksMD

Indicates article with supplemental on-line appendix.

Indicates article with supplemental on-line photo.

http://dx.doi.org/10.3174/ajnr.A6429 
A fundamental factor in patient outcomes from DBS is related to appropriate targeting and device programming; however, intraoperative macrostimulation and subsequent programming are problematic in dystonia due to the typical delay in symptom improvement (days-weeks) compared with the near-immediate effect in some other movement disorders (eg, Parkinson disease and essential tremor). ${ }^{8}$ Thus, establishment of accurate markers for targeting and programming is an imperative step to maximize outcomes of patients with dystonia undergoing DBS.

The role of MR imaging-derived connectivity measures from fMRI and DTI has been increasingly explored in DBS for movement disorders, particularly for essential tremor and Parkinson disease. ${ }^{9-13}$ While functional neurosurgical targeting has historically relied heavily on coordinate-based stereotaxy using easily identified landmarks (eg, anterior/posterior commissure [ACPC] line), such fixed anatomic coordinate systems have been shown to not account for patient-specific anatomic and network differences, yielding significant variability with respect to the underlying brain being impacted by neuromodulation. ${ }^{14}$ Thus, ACPC coordinates of active DBS contacts have previously failed to correlate with outcomes in dystonia. ${ }^{15}$

On the basis of the predictability of outcomes from fMRI and DTI measures shown in previous studies of DBS in other movement disorders, we hypothesized that connectivity data could be used as an independent biomarker of GPi DBS success in inherited or idiopathic-isolated dystonia (formerly, primary dystonia). ${ }^{9,10,13}$ In this study, we evaluated the functional and structural connectivity patterns that correlate with successful outcomes in GPi DBS for inherited or idiopathic-isolated generalized dystonia.

\section{MATERIALS AND METHODS}

A retrospective analysis of the INFORM Patient Research Database (Norman Fixel Institute for Neurological Diseases, University of Florida, Gainesville, Florida) was approved by the University of Florida institutional review board. Patients meeting the following criteria were placed with the corresponding Unified Dystonia Rating Scale (UDRS) score: 1) diagnosis of inherited or idiopathic-isolated generalized dystonia according to consensus criteria, ${ }^{1}$ diagnosed by a movement disorder neurologist with a preoperative UDRS score; 2) simultaneous or staged DBS of the bilateral GPi; 3) absence of other brain operations or prior DBS; 4) preoperative MR imaging and postoperative CT; and 5) $\geq 6$-month postoperative follow-up after both electrodes were placed. The primary end point was defined as percentage improvement in the UDRS from the preoperativeto-postoperative follow-up UDRS. The patients' charts and programming data were retrospectively reviewed, and we chose the time point when the DBS settings were considered optimum (range, 6-24 months).

\section{Patient Selection and Surgical Procedure}

The DBS candidacy was decided after a consensus review at a multidisciplinary conference, including neurology, neurosurgery, psychiatry, neuropsychology, and rehabilitation medicine. Patients were evaluated monthly for the first 6 postoperative months to optimize stimulation settings, followed by biannual visits. Nonresponders were defined as having achieved $\leq 25 \%$ improvement in the UDRS postoperatively, consistent with a previously published classification. ${ }^{4,15,16}$

\section{Image Processing}

Electrode localization and stimulation of volumes of tissue activated (VTAs) were performed in the LEAD-DBS software package (http://www.lead-dbs.org). ${ }^{17}$ Full details of imaging preprocessing and electrode localization can be found in the On-line Appendix.

\section{Functional Connectivity}

VTAs were generated from each patient's most effective programming settings, as described in Horn et al. ${ }^{9}$ All right-hemisphere VTAs underwent a nonlinear mirroring to the left side. Each left hemisphere and mirrored right-hemisphere VTA then served as a seed point for functional connectivity measures. Functional connectivity data were estimated from 1000 healthy subjects in the Brain Genomics Superstruct Project (https://dataverse.harvard.edu/dataverse/GSP) ${ }^{18}$ using previously described methods. ${ }^{9}$ Details of the fMRI acquisition and preprocessing are presented in the On-line Appendix. After correlation between the VTA seed and all brain voxels, the resultant r-maps underwent Fisher z-transformation. The individual connectivity maps were then correlated with improvement in the UDRS and controlled for age and stimulation frequency using a general linear model, as implemented in Statistical Parametric Mapping (SPM; Version 12; https://www.fil.ion.ucl.ac.uk/spm/). Maps for positive and negative correlation with UDRS were generated and thresholded at a $t$-score corresponding to $P<.05$.

\section{Structural Connectivity}

The left-hemisphere and mirrored right-hemisphere VTAs were used as seed volumes for structural connectivity as implemented in LEAD-DBS. The structural connectivity was estimated on the basis of a normative dataset of 32 healthy subjects in the Human Connectome Project imaged at Massachusetts General Hospital (https://ida.loni.usc.edu/login.jsp). Resultant structural connectivity maps underwent a voxelwise correlation analysis using SPM, Version 12. A general linear model was used to assess positive and negative correlations with the percentage of UDRS improvement, controlled for age and stimulation frequency. The resulting $t$-maps were thresholded to $P<.05$.

\section{VTA Analysis}

Group-level analysis of the normalized VTAs was then performed. Each VTA was associated with the corresponding improvement in the UDRS score, and a 2-sample $t$ test was used to assign a $t$-score to each voxel, which rendered a "heat map" of the treatment response. Both positive and negative correlations were assessed to compare with previously published results of spatial correlation with outcomes. ${ }^{15}$ Clusters were assessed for peak $t$-score and center of gravity to determine the spatial localization of peak improvement in the UDRS percentage change using the "cluster" function of FSL (Version 6; https://fsl.fmrib. ox.ac.uk/fsl/fslwiki/). VTAs for responders and nonresponders 


\begin{tabular}{|c|c|c|c|c|c|}
\hline \multirow[b]{2}{*}{ Characteristic } & & \multicolumn{4}{|c|}{ DBS Programming Information } \\
\hline & & \multicolumn{2}{|l|}{ Left } & \multicolumn{2}{|l|}{ Right } \\
\hline Age at surgery (yr) & $42.9(7-83)$ & Frequency $(\mathrm{Hz})$ & $105.8( \pm 45.1)$ & Frequency $(\mathrm{Hz})$ & $108.1( \pm 45.5)$ \\
\hline $\operatorname{Sex}(M / F)$ & $21: 18$ & Pulse width ( $\mu$ s) & $216.9( \pm 99.4)$ & Pulse width ( $\mu$ s) & $219.8( \pm 110.9)$ \\
\hline Age of onset (yr) & $31.1(2-65)$ & Voltage (V) & $2.9( \pm 0.7)$ & Voltage (V) & $2.8( \pm 0.6)$ \\
\hline Disease duration before DBS (yr) & $14.3( \pm 14.0)$ & Impedance $(\Omega ; n=35)$ & $1066.6( \pm 355.0)$ & Impedance $(\Omega ; n=35)$ & $1129.5( \pm 532.6)$ \\
\hline Follow-up period after DBS (mo) & $13.4( \pm 6.6)$ & No. (\%) leads in & $17(43.6 \%)$ & No. (\%) leads in & $19(48.7 \%)$ \\
\hline Positive for DYT7 mutation & 9 & bipolar mode ${ }^{a}$ & & bipolar mode $\mathrm{a}^{\mathrm{a}}$ & \\
\hline Baseline UDRS & $33( \pm 23.3)$ & & & & \\
\hline Postoperative UDRS & $14.6( \pm 15)$ & & & & \\
\hline UDRS improvement (\%) & $53.2 \%( \pm 34.5 \%)$ & & & & \\
\hline $\begin{array}{l}\text { Nonresponders (No.) (\%) } \leq 25 \% \\
\text { improvement }\end{array}$ & $7(17.9 \%)$ & & & & \\
\hline
\end{tabular}

a Patients programmed with bilateral bipolar configuration $(n=13)$.

were averaged separately, and the center of gravity for the resulting VTA clusters was calculated.

Coordinates from the current study and previous studies ${ }^{15,19-21}$ were transformed between ACPC space and Montreal Neurological Institute (MNI) template space using a probabilistic transformation $^{22}$ based on nonlinear deformation fields of 14 patients with dystonia (age range, 7-65 years), as implemented in LEADDBS software, to compare with previously reported spatial coordinates of maximal GPi DBS efficacy in dystonia.

VTAs were also assessed for the volume of overlap with the $\mathrm{GPi}$, as defined in the DBS Intrinsic Template Atlas ${ }^{23}$ and correlated with the percentage change in the UDRS. A 2-tailed $t$ test was also performed to compare the normalized volume of overlap between the GPi and VTA in responders versus nonresponders.

\section{Statistical Analysis}

Demographic and DBS programming data were expressed as mean and SD. The Mann-Whitney test was used to compare differences in demographics and DBS programming data between responders and nonresponders using GraphPad Prism, Version 8.1.1 (GraphPad Software, San Diego, California). A $P$ value $<.05$ was considered statistically significant.

\section{RESULTS}

A total of 43 patients meeting the inclusion criteria were identified. Four were excluded due to inadequate imaging quality and resultant suboptimal electrode localization, leaving a total of 39 subjects. Demographic data, clinical outcomes, and DBS programming parameters are summarized in Table 1 . Active contact positions relative to the GPi are illustrated in Fig $1 A-C$. Of 78 total leads, $71.8 \%$ $(n=56)$ had at least 1 of the 2 dorsal contacts $(\mathrm{k} 2 / \mathrm{k} 3)$ activated. There was no correlation between VTA overlap with the GPi and UDRS improvement $\left(r^{2}=0.05 ; P=.4\right)$ (Fig 1D).

Seven patients $(17.9 \%)$ were classified as nonresponders ( $\leq 25 \%$ UDRS improvement). There was no significant difference in age of onset, age at the operation, duration of follow-up, or disease duration between responders and nonresponders (all, $P>$.13). There was no significant difference in voltage, frequency, pulse width, impedance, or number of bipolar configurations used (all $P>.1$ ). There was no significant difference in the volume of overlap between the GPi and VTA in responders versus nonresponders $(P=.32)$. Additionally, the center of gravity for the averaged VTA clusters was identical between the responders and nonresponders $(\mathrm{MNI}=-24 /-9 /-3)$.

Assessment of VTA regions correlating with UDRS improvement (Fig 2) showed the highest correlation in the ventrolateral GPi along its border with the globus pallidus externus (cluster center-of-gravity MNI: right $=23.5 /-7 /-3$ and left $=$ $-24.5 /-8 /-3.5)$. Peak $t$-scores for the positive clusters were 2.86 on the right and 3.14 on the left. Two significant clusters were identified on each side, corresponding to a negative correlation with UDRS improvement. On the left, there was 1 cluster center of gravity at MNI $=-25 /-9.5 / 2$ with a peak $t$-score of -3.07 and a second at $\mathrm{MNI}=-22 /-4.5 /-4.5$ with a peak $t$-score of -2.87 . On the right, there was 1 cluster center of gravity at MNI $=23 /-5.5 / 2$, with a peak $t$-score of -2.46 and a second at MNI $=$ $22.5 /-12 /-4.5$ with a peak $t$-score of -2.58 . The cluster most correlated with UDRS improvement corresponded to an estimated spheric VTA with a $3-\mathrm{mm}$ radius centered at $\mathrm{MNI}=$ $-24 /-10.2 /-3.7$, which showed a similar connectivity pattern to the regions correlated with greater UDRS score improvement (On-line Figure).

Structural connectivity correlating with the UDRS score (Fig $3 A$ ) showed multiple areas predictive of UDRS improvement, such as along the dorsal primary motor cortex corresponding to areas responsible for motor function of the trunk, arm, and hand. Additional areas of connectivity correlating with UDRS improvement were present in the lateral frontal, inferior parietal, and lateral temporo-occipital lobes. Structural connectivity correlated with worse UDRS improvement was greatest in the medial prefrontal region.

Functional connectivity (Fig $3 B$ ) was strongly correlated with improved UDRS scores in many nodes of the motor network, including the primary sensorimotor regions, motor thalamus (Fig $3 C$ ), and cerebellum (Fig 3D). There was also a positive correlation with the superior temporal gyrus and lateral occipital lobe. A negative correlation with UDRS improvement was present in multiple areas, including the supplemental motor area, lateral occipital, inferior temporal, and superior, middle, and inferior frontal gyri.

\section{DISCUSSION}

In this study, we have demonstrated the functional and structural connectivity patterns associated with clinical improvement in generalized dystonia after DBS. Although the pathophysiology of 

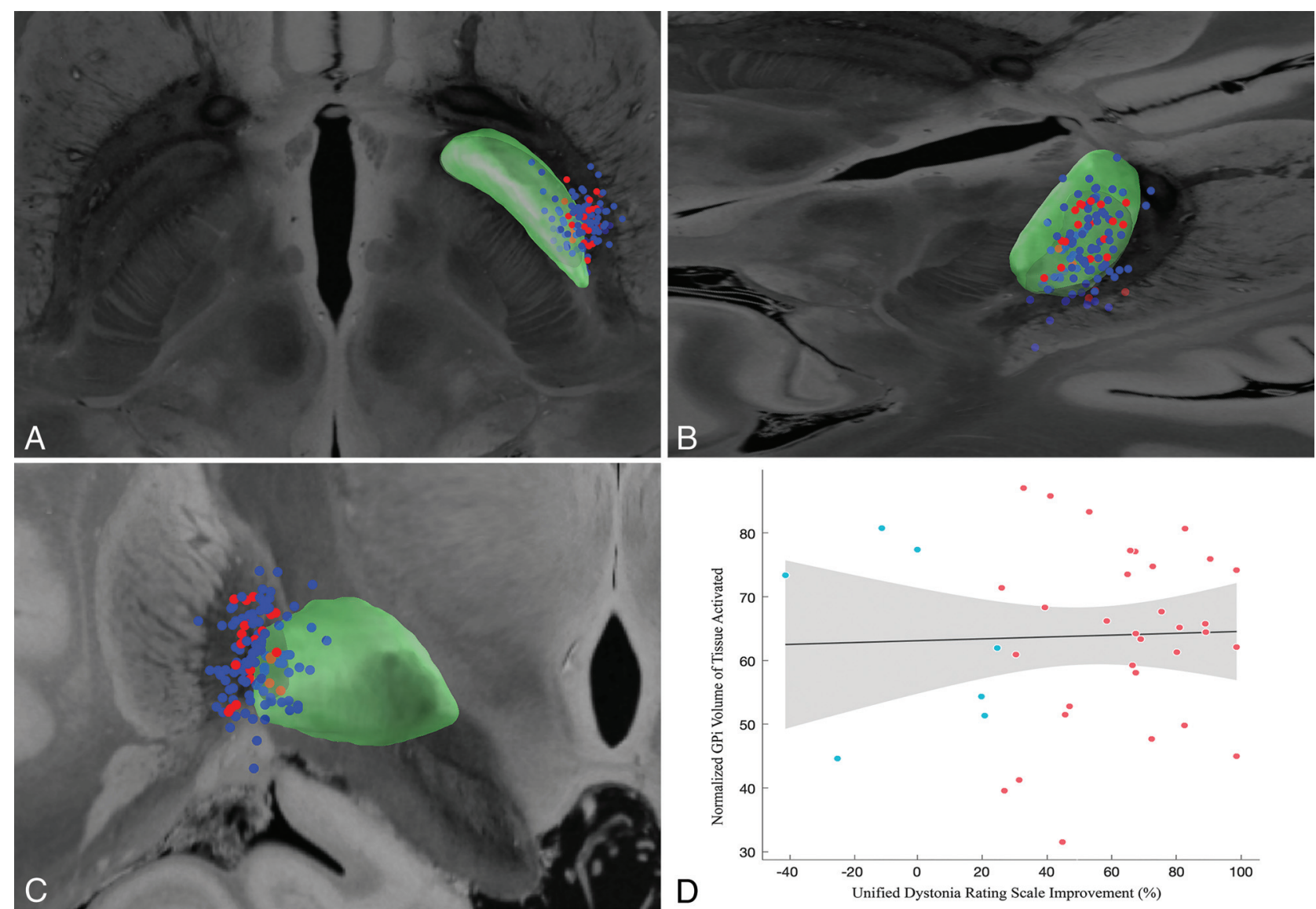

FIG 1. Axial $(A)$, posterior oblique $(B)$, and coronal $(C)$ views of the position of the active contacts for all subjects relative to the globus pallidus internus (green). Contacts for nonresponders ( $\leq 25 \%$ improvement) are shown as red and responders as blue. All active contacts are nonlinearly mirrored to the same side. $D$, Normalized volume of tissue activated overlapping the globus pallidus internus and the Unified Dystonia Rating Scale improvement shows no significant correlation $\left(r^{2}=0.05 ; P=.4\right)$.
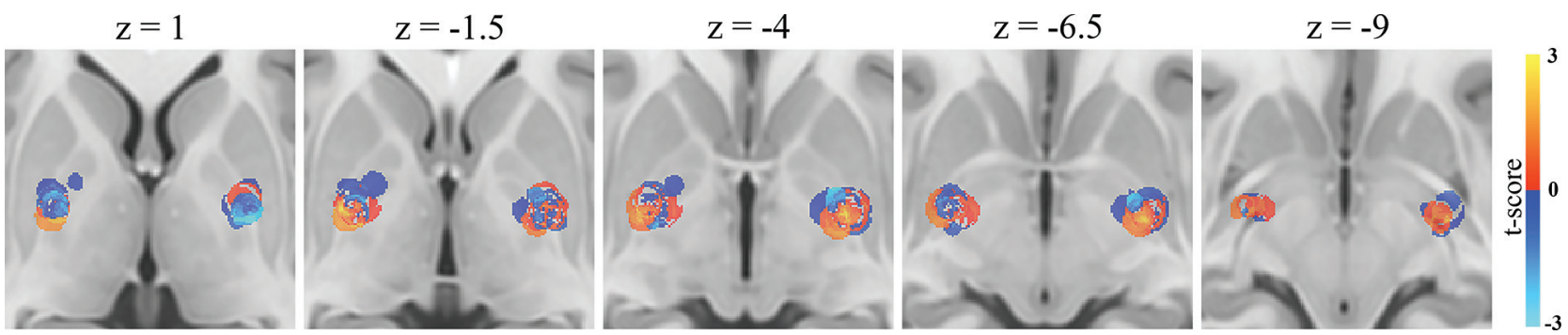

FIG 2. Heat maps showing spatial correlation of volumes of tissue activated positively (red) and negatively (blue) correlated with improvement in the Unified Dystonia Rating Scale score.

inherited or idiopathic-isolated dystonia is poorly understood, existing studies have revealed disturbances within multiple nodes of the motor network. ${ }^{24-26}$ The results of our study are concordant with earlier findings showing a positive correlation between motor improvement and connectivity to the primary sensorimotor cortex, motor thalamus, and cerebellum. ${ }^{24,27}$

DBS has been shown to be an effective treatment for dystonia; however, nonresponder rates of up to $25 \%$ have been reported. ${ }^{28}$ This variability may be due to multiple factors, such as the age at disease onset, dystonia severity, genetic status, skeletal deformities, and lead location. ${ }^{15,28-30}$ Genetic factors may also play a role. For example, patients having the TOR1A (DYT1) mutation-often leading to appendicular phenomenology at presentation-have been described as benefitting more from DBS treatment in comparison with patients with THAP1 (DYT6), which often presents with cranio-cervico-laryngeal symptoms. ${ }^{28}$ Furthermore, no consensus has been reached regarding screening procedures, training requirements for treatment providers, and patient management guidelines for complications. ${ }^{31}$ In contrast to other common movement disorders treated with DBS, 1 problematic feature of dystonia DBS treatment is the delay in therapeutic response, which is typically observed after days-months following the adjustment of stimulation. ${ }^{32}$ The lack of near-instantaneous feedback of the treatment response to a particular 

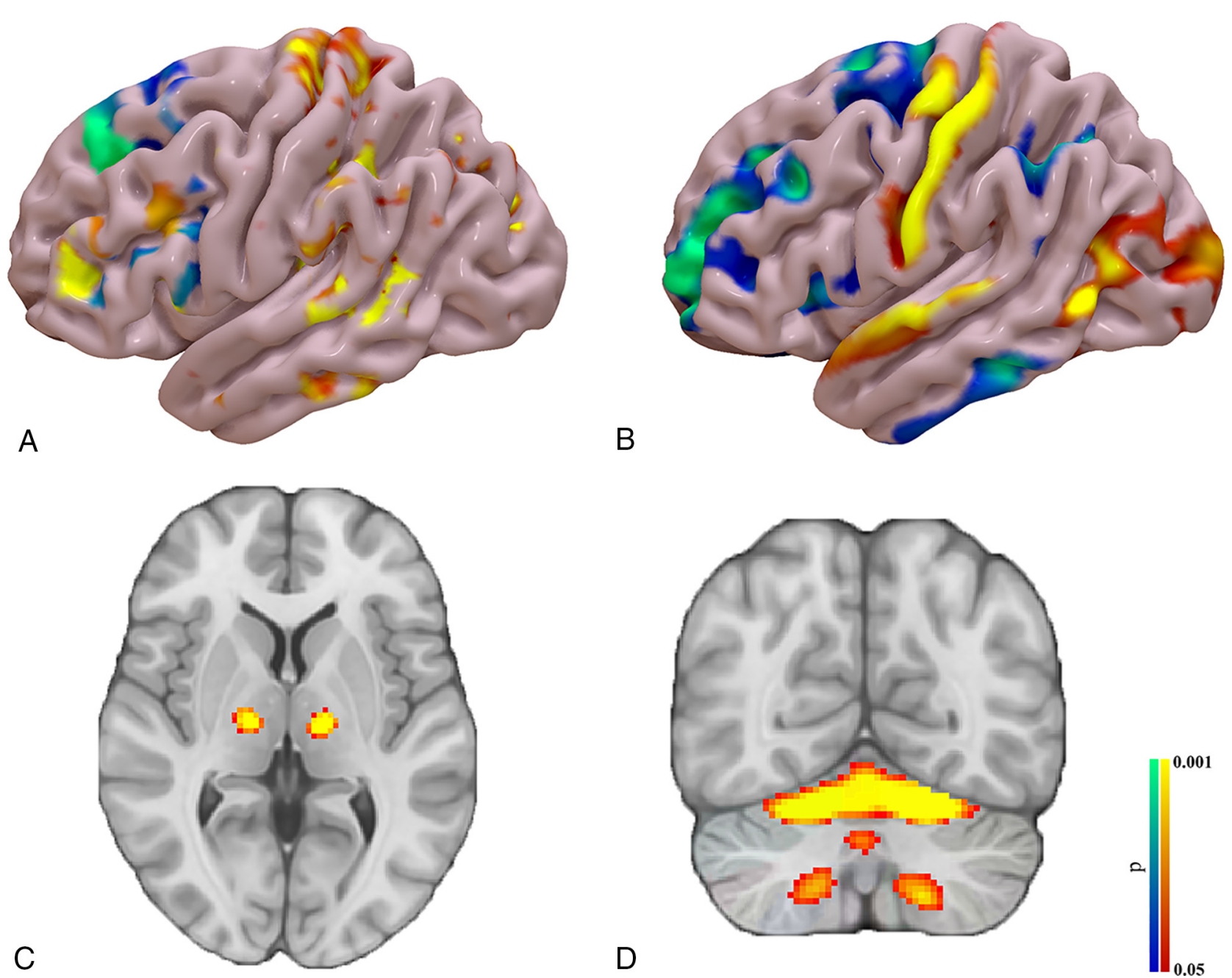

B

FIG 3. (A) Group-level structural connectivity (DTI) results showing cortical connections positively (red) and negatively (blue) correlated with improvement in the UDRS score. Resting-state functional connectivity results show cortical $(B)$, subcortical $(C)$, and cerebellar $(D)$ connectivity profiles correlated with improvement in the UDRS score.

stimulation setting limits accurate device programming. ${ }^{32}$ The summation of these findings highlights the complexity of achieving maximal therapeutic benefit for dystonia with DBS and the necessity for new biomarkers.

DBS targeting accuracy has historically been assessed primarily as a function of the electrode contact position relative to the target nucleus; however, Reich et $\mathrm{al}^{15}$ have shown the poor predictability of such rudimentary measures on outcomes for movement disorders. In our study, we observed no distinct correlation between the relationship of the electrode position relative to the GPi. While assessment of VTA may be a more predictive feature of DBS outcome, overall VTA overlap with the GPi was not predictive of UDRS improvement. Heat maps of VTA overlap and UDRS improvement, however, did reveal a "sweet spot" of stimulation lying along the ventrolateral GPi along its border with the globus pallidus externus. Our findings were concordant with those reported by other groups, as summarized in Table $2 .{ }^{15,19-21}$ Minor variations in ideal coordinates for the anti-dystonic effect are potentially related to differences in the phenomenology of the sample, the surgical targeting technique, and the actual stimulation settings.
Recent DTI studies of the human pallidum have suggested a more complex structural connectivity pattern than considered initially. ${ }^{13,27,33-35}$ In particular, a bipartite pattern of connectivity within the posterior "sensorimotor" pallidum from dorsal to ventral has been shown and may be a predictor of DBS outcome in various movement disorders. ${ }^{13}$ Rozanski et $\mathrm{al}^{27}$ had shown, in a small cohort with focal dystonia, that greater structural connectivity to more posterior cortical regions, including the primary motor and sensory cortices, correlated with a greater anti-dystonic effect, while more anterior motor and premotor regions were associated with less clinical improvement. Our results show similar findings within a larger generalized dystonia population undergoing DBS. Specifically, greater connectivity with the ipsilateral medial peri-Rolandic region correlated with greater motor improvement, while more anterior prefrontal connectivity was inversely correlated with motor improvement.

In a recent study of network connectivity associated with acquired cervical dystonia from brain lesions, Corp et $\mathrm{al}^{24}$ found that lesions with connectivity to lobule IX of the cerebellum and the somatosensory cortex had the greatest sensitivity and specificity for development of cervical dystonia. When examining the 
Table 2: Comparison of stereotactic coordinates from prior studies reporting maximum anti-dystonic effect

\begin{tabular}{|c|c|c|c|c|c|c|}
\hline & \multicolumn{3}{|c|}{ MNI Coordinate (mm) } & \multicolumn{3}{|c|}{ ACPC Coordinate $(\mathrm{mm})$} \\
\hline & $\mathbf{x}$ & $y$ & z & $\mathbf{x}$ & $y$ & z \\
\hline Current Study & -24 & -7.5 & -3.25 & -21.9 & 3.6 & -0.2 \\
\hline Cheung et $\mathrm{al}^{19}$ & -22.1 & -8 & -2.5 & -20.2 & 3 & 0.7 \\
\hline Reich et $\mathrm{al}^{15}$ & -19.4 & -10.1 & -5.9 & -19.4 & 3.2 & -1.9 \\
\hline Starr et $\mathrm{al}^{20 \mathrm{a}}$ & -21.6 & -5.5 & -4.3 & -19.8 & 5.6 & -0.6 \\
\hline Pauls et $\mathrm{al}^{21}$ & -22.9 & -7.2 & -5.9 & -20.9 & 4.1 & -2.1 \\
\hline
\end{tabular}

a Patients with $>70 \%$ Burke-Fahm-Marsden Dystonia Rating Scale improvement.

connectivity pattern within these 2 brain regions from a cohort of good and poor DBS responders with dystonia, good responders exhibited a greater positive correlation with the cerebellum and greater negative correlation with the somatosensory cortex. ${ }^{24}$ In our generalized dystonia cohort, we found a similar pattern of DBS connectivity correlating with clinical improvement, including the cerebellum and somatosensory cortex; however, we also found positive and negative correlations in multiple areas outside those interrogated by Corp et al. ${ }^{24}$

Our results revealed connectivity correlations with multiple areas outside the primary somatosensory and cerebellar regions, including the supplementary motor area, prefrontal cortex, inferior parietal lobule, lateral temporal, and lateral occipital regions. Most interesting, many of these areas were noted to exhibit cortical thickness differences between patients with poor response versus good response to GPi DBS for segmental and generalized dystonia in a recent study by Gonzalez-Escamilla et al. ${ }^{36}$ Using graph theory, their study posited that changes in centrality and the clustering coefficient in these prefrontal, parietal, temporal, and lateral occipital regions suggest that they may be a cause of network failure and decreased DBS effectiveness. ${ }^{36}$ Our findings of negative functional connectivity correlation with UDRS improvement in many of these regions support the idea that they may play a role in limiting the neuromodulatory effect of GPi DBS for dystonia.

Several limitations of our study are noteworthy. The use of normative connectomes is a common practice in DBS research due to the inherent difficulty of obtaining such information in a large cohort of patients with movement disorders. Nevertheless, it is currently unknown whether such normative data are directly applicable to individual patients. Additionally, the variation in stimulation parameters, such as frequency and pulse width, can further complicate the understanding of the mechanism of action for DBS. While we have attempted to account for these variations in our study design, it is uncertain whether these findings are directly applicable to patients undergoing different stimulation parameters. Finally, interrater agreement of UDRS assessments was fair to good in some body regions, as is the case with the Burke-Fahn-Marsden Dystonia Rating Scale. ${ }^{37}$ Additionally, the UDRS and Burke-Fahn-Marsden Dystonia Rating Scale allow clinicians to assess overall dystonia severity but may be less sensitive for cervical dystonia assessments compared with the Toronto Western Spasmodic Torticollis Rating Scale. ${ }^{38}$ Better clinometric scales are warranted in future studies.

\section{CONCLUSIONS}

DBS is a promising therapy for patients with generalized dystonia; however, its applicability is currently limited by delays in achieving a reproducible and optimal therapeutic outcome and the lack of an acute bedside marker of the success of DBS programming. The major challenge in dystonia DBS is the determination of an ideal stimulation parameter setting due to the delay in appearance of improvement in motor symptoms compared with DBS in other common movement disorders. The development of an accurate marker may help maximize treatment outcomes. We have shown a pattern of structural and functional connectivity associated with clinical improvement in generalized dystonia that may serve as a basis for an imaging marker for DBS programming. Additional studies will be required to determine the clinical applicability of our model.

\section{ACKNOWLEDGMENTS}

The authors acknowledge the assistance of Lucy Bahn, PhD, in preparation of this article.

Disclosures: Robert S. Eisinger-UNRELATED: Grants/Grants Pending: National Institute of Neurological Disorders and Stroke F30 Award. Leonardo AlmeidaUNRELATED: Consultancy: Medtronic, Boston Scientific, Comments: I have worked for and received honoraria from Medtronic and Boston Scientific as a consultant for the Scientific and Product Advisory Board. Kelly Foote-UNRELATED: Consultancy: Medtronic, Boston Scientific, Comments: occasional paid consultant for deep brain stimulation-related issues; Grants/Grants Pending: Medtronic, Boston Scientific, Functional Neuromodulation, Comments: Medtronic, research support (investigational devices and engineering support) provided for multiple investigator-initiated closedloop deep brain stimulation research projects; Medtronic, grant for partial support of a functional neurosurgery fellowship at the University of Florida; Boston Scientific and Functional Neuromodulation, grant support to our institution for participation in industry-sponsored deep brain stimulation research (Parkinson and Alzheimer diseases)*; Payment for Development of Educational Presentations: Medtronic, Comments: support paid to the University of Florida in exchange for education provided to visiting deep brain stimulation practitioners.* Michael S. Okun—RELATED: Grant: National Institutes of Health and Parkinson's Foundation, Comments: I have grants from the National Institutes of Health and Parkinson's Foundation but not directly attached to this work*; UNRELATED: Consultancy: Parkinson's Foundation, Comments: Medical Director. ${ }^{\star}$ Money paid to the institution.

\section{REFERENCES}

1. Albanese A, Bhatia K, Bressman SB, et al. Phenomenology and classification of dystonia: a consensus update. Mov Disord 2013;28:863-73 CrossRef Medline

2. Jahanshahi M, Czernecki V, Zurowski AM. Neuropsychological, neuropsychiatric, and quality of life issues in DBS for dystonia. Mov Disord 2011;26(Suppl 1):S63-S78 CrossRef Medline

3. Vidailhet M, Vercueil L, Houeto JL, et al; French SPIDY Study Group. Bilateral, pallidal, deep-brain stimulation in primary generalised dystonia: a prospective 3 year follow-up study. Lancet Neurol 2007;6:223-29 CrossRef Medline

4. Volkmann J, Wolters A, Kupsch A, et al. Pallidal deep brain stimulation in patients with primary generalised or segmental dystonia: 5 year follow-up of a randomised trial. Lancet Neurol 2012;11:102938 CrossRef Medline 
5. Moro E, LeReun C, Krauss JK, et al. Efficacy of pallidal stimulation in isolated dystonia: a systematic review and meta-analysis. Eur J Neurol 2017;24:552-60 CrossRef Medline

6. Vidailhet M, Vercueil L, Houeto JL, et al; French Stimulation du Pallidum Interne dans la Dystonie (SPIDY) Study Group. Bilateral deep-brain stimulation of the globus pallidus in primary generalized dystonia. N Engl J Med 2005;352:459-67 CrossRef Medline

7. Volkmann J, Mueller J, Deuschl G, et al; DBS Study Group for Dystonia. Pallidal neurostimulation in patients with medicationrefractory cervical dystonia: a randomised, sham-controlled trial. Lancet Neurol 2014;13:875-84 CrossRef Medline

8. Hu W, Stead M. Deep brain stimulation for dystonia. Transl Neurodegener 2014;3:2 CrossRef Medline

9. Horn A, Reich M, Vorwerk J, et al. Connectivity predicts deep brain stimulation outcome in Parkinson disease. Ann Neurol 2017;82:6778 CrossRef Medline

10. Akram H, Dayal V, Mahlknecht P, et al. Connectivity derived thalamic segmentation in deep brain stimulation for tremor. Neuroimage Clin 2018;18:130-42 CrossRef Medline

11. Middlebrooks EH, Grewal SS, Stead M, et al. Differences in functional connectivity profiles as a predictor of response to anterior thalamic nucleus deep brain stimulation for epilepsy: a hypothesis for the mechanism of action and a potential biomarker for outcomes. Neurosurg Focus 2018;45:E7 CrossRef Medline

12. Middlebrooks EH, Holanda VM, Tuna IS, et al. A method for preoperative single-subject thalamic segmentation based on probabilistic tractography for essential tremor deep brain stimulation. Neuroradiology 2018;60:303-09 CrossRef Medline

13. Middlebrooks EH, Tuna IS, Grewal SS, et al. Segmentation of the globus pallidus internus using probabilistic diffusion tractography for deep brain stimulation targeting in Parkinson disease. AJNR Am J Neuroradiol 2018;39:1127-34 CrossRef Medline

14. Middlebrooks EH, Tuna IS, Almeida L, et al. Structural connectivity-based segmentation of the thalamus and prediction of tremor improvement following thalamic deep brain stimulation of the ventral intermediate nucleus. Neuroimage Clin 2018;20:1266-73 CrossRef Medline

15. Reich MM, Horn A, Lange F, et al. Probabilistic mapping of the antidystonic effect of pallidal neurostimulation: a multicentre imaging study. Brain 2019;142:1386-98 CrossRef Medline

16. Tsuboi T, Jabarkheel Z, Foote KD, et al. Importance of the initial response to GPi deep brain stimulation in dystonia: a nine year quality of life study. Parkinsonism Relat Disord 2019;64:249-55 CrossRef Medline

17. Horn A, Li N, Dembek TA, et al. Lead-DBS v2: towards a comprehensive pipeline for deep brain stimulation imaging. Neuroimage 2019;184:293-316 CrossRef Medline

18. Buckner RL, Roffman JL, Smoller JW. Brain Genomics Superstruct Project (GSP) Dataverse. 2014. Harvard Dataverse, V10 CrossRef

19. Cheung T, Noecker AM, Alterman RL, et al. Defining a therapeutic target for pallidal deep brain stimulation for dystonia. Ann Neurol 2014;76:22-30 CrossRef Medline

20. Starr PA, Turner RS, Rau G, et al. Microelectrode-guided implantation of deep brain stimulators into the globus pallidus internus for dystonia: techniques, electrode locations, and outcomes. J Neurosurg 2006;104:488-501 CrossRef Medline
21. Pauls KA, Brockelmann PJ, Hammesfahr S, et al. Dysarthria in pallidal deep brain stimulation in dystonia depends on the posterior location of active electrode contacts: a pilot study. Parkinsonism Relat Disord 2018;47:71-75 CrossRef Medline

22. Horn A, Kuhn AA, Merkl A, et al. Probabilistic conversion of neurosurgical DBS electrode coordinates into MNI space. Neuroimage 2017;150:395-404 CrossRef Medline

23. Ewert S, Plettig P, Li N, et al. Toward defining deep brain stimulation targets in MNI space: a subcortical atlas based on multimodal MRI, histology and structural connectivity. Neuroimage 2018;170:271-82 CrossRef Medline

24. Corp DT, Joutsa J, Darby RR, et al. Network localization of cervical dystonia based on causal brain lesions. Brain 2019;142:1660-74 CrossRef Medline

25. Balint B, Mencacci NE, Valente EM, et al. Dystonia. Nat Rev Dis Primers 2018;4:25 CrossRef Medline

26. Jinnah HA, Neychev V, Hess EJ. The anatomical basis for dystonia: the motor network model. Tremor Other Hyperkinet Mov (N Y) 2017;7:506 CrossRef Medline

27. Rozanski VE, Vollmar C, Cunha JP, et al. Connectivity patterns of pallidal DBS electrodes in focal dystonia: a diffusion tensor tractography study. Neuroiimage 2014;84:435-42 CrossRef Medline

28. Lozano AM, Lipsman N, Bergman H, et al. Deep brain stimulation: current challenges and future directions. Nat Rev Neurol 2019;15:148-60 CrossRef Medline

29. Isaias IU, Alterman RL, Tagliati M. Outcome predictors of pallidal stimulation in patients with primary dystonia: the role of disease duration. Brain 2008;131:1895-902 CrossRef Medline

30. Pauls KA, Krauss JK, Kampfer CE, et al. Causes of failure of pallidal deep brain stimulation in cases with pre-operative diagnosis of isolated dystonia. Parkinsonism Relat Disord 2017;43:38-48 CrossRef Medline

31. Okun MS, Tagliati M, Pourfar M, et al. Management of referred deep brain stimulation failures: a retrospective analysis from 2 movement disorders centers. Arch Neurol 2005;62:1250-55 CrossRef Medline

32. Reese R, Volkmann J. Deep brain stimulation for the dystonias: evidence, knowledge gaps, and practical considerations. Mov Disord Clin Pract 2017;4:486-94 CrossRef Medline

33. Grewal SS, Holanda VM, Middlebrooks EH. Corticopallidal connectome of the globus pallidus externus in humans: an exploratory study of structural connectivity using probabilistic diffusion tractography. AJNR Am J Neuroradiol 2018;39:2120-25 CrossRef Medline

34. Cacciola A, Calamuneri A, Milardi D, et al. A connectomic analysis of the human basal ganglia network. Front Neuroanat 2017;11:85 CrossRef Medline

35. Cacciola A, Milardi D, Quartarone A. Role of cortico-pallidal connectivity in the pathophysiology of dystonia. Brain 2016;139:e48 CrossRef Medline

36. Gonzalez-Escamilla G, Muthuraman M, Reich MM, et al. Cortical network fingerprints predict deep brain stimulation outcome in dystonia. Mov Disord 2019;34:1536-45 CrossRef Medline

37. Comella CL, Leurgans S, Wuu J, et al; Dystonia Study Group. Rating scales for dystonia: a multicenter assessment. Mov Disord 2003;18:303-12 CrossRef Medline

38. Albanese A, Sorbo FD, Comella C, et al. Dystonia rating scales: critique and recommendations. Mov Disord 2013;28:874-83 CrossRef Medline 